How do People Judge the Immorality of Artificial Intelligence versus Humans Committing Moral Wrongs in Real-World Situations?

\author{
Abigail Wilson, Courtney Stefanik, and Daniel B. Shank \\ Department of Psychological Science, Missouri University of Science and Technology \\ Preprint Under Review - This may not be the final version.
}

\begin{abstract}
Author Note
Abigail Wilson https://orcid.org/0000-0002-9389-2217

Courtney Stefanik https://orcid.org/0000-0002-1984-0603

Daniel B. Shank https://orcid.org/0000-0002-3746-2407
\end{abstract}

We have no known conflict of interest to disclose. This study was supported by the First Year Research Experience program at Missouri University of Science and Technology as well as the Army Research Office under Grant Number W911NF-19-1-0246.

Correspondence concerning this article should be addressed to Daniel B. Shank, Department of Psychological Science, $500 \mathrm{~W} 14^{\text {th }}$ St, Rolla, MO, 65401. Email:

shankd@mst.edu 


\begin{abstract}
In general, people will judge a morally wrong behavior when perpetrated by an artificial intelligence (AI) as still being wrong. But moral judgements are complex, and therefore we explore how moral judgements made about AIs differ from those made about humans in realworld situations. In contrast to much of the current research on the morality of AIs, we examine real-world encounters where an AI commits a moral wrong as reported by participants in previous research. We adapt these to create nearly identical scenarios with human perpetrators. In Study 1, across scenarios, humans are perceived as more wrong, intentional, and to blame compared to AIs. In Study 2, we replicate those results and find that by showing the participants the contrasting scenario - showing the AI scenario when one is rating the human scenario or vice versa - does not have a significant effect on moral judgements. An exploratory word-frequency analysis and illustrative quotes from participants' open-ended explanations show that AIs are more often denied agency and perceived as programmed and therefore unintentional in producing the moral outcome. In contrast, humans are perceived as highly agentic and intentional, either fully responsible for the wrongdoing or not morally culpable because the behavior was perceived as a mistake. Keywords: Artificial Intelligence, Algorithms, Machines, Moral Judgment, Agency
\end{abstract}




\section{How do People Judge the Immorality of Artificial Intelligence versus Humans Committing Moral Wrongs in Real-World Situations?}

Traditionally, artificial intelligence (AI) has been used to assist humans in the completion of tasks. As technology has become more advanced, so have the capabilities of AI. Jobs that were once reserved solely for humans are now being completed by AIs. Robots and AI can drive cars, provide care for patients, and even approve or deny individuals applying for welfare benefits (Waytz et al., 2014; Van Wynsberghe, 2012; Eubanks, 2019). AIs in these domains may make decisions which have moral consequences and commit acts that would be judged as morally wrong if done by a human. For example, there are currently 23 accidents being investigated by the National Highway Traffic Safety Administration that have all been linked with Tesla's self-driving cars, with at least three of these accidents being fatal (Boudette, 2021). How do people judge an AI for committing a moral wrong compared to humans committing that same moral wrong?

Although humans are interacting more with AI, there has been little research studying morality in real-world scenarios. Past research often uses moral dilemmas such as the trolley dilemma, which involves a moral actor being forced to choose between non-action leading to multiple people's deaths versus action which save that group but leads to the death of a single individual (Malle et al., 2015; Voiklis et al., 2016). These studies then ask participants to make judgments on blame and morality (Malle et al., 2015; Voiklis et al., 2016). Moral dilemma research design only allows for one moral violation, harm, to be studied in the context of humanAI interaction. In contrast, our research focuses on the use of real-world encounters with AI. These scenarios used herein were collected from past research in which individuals reported events they personally encountered or witnessed where an AI committed a moral wrong (Shank 
$\&$ Gott, 2019). The use of a large number of real-world scenarios allows us to understand the judgments people make after various moral violations, none of which are the life and death scenarios of most moral dilemmas (Shank \& Gott, 2020).

\section{Types of Moral Judgments}

When a moral dilemma has occurred, questions of both moral permissibility and responsibility arise. A morally permissible action is one that is not deemed as morally wrong, but rather something that is acceptable and can be justified. While an individual may not be faulted for their actions, this does not mean that the action was not morally wrong, as wrongness is the focus on the behavior rather than on the agent (Malle et al., 2014). Murder, for example, is held as a moral wrong. While the behavior itself has a moral judgment, separate judgments are made for the person committing the action. Moral responsibility looks at who or what is responsible for actions and outcomes (Wegner \& Gray, 2017). Additionally, the agent committing the behavior can be judged as to whether their actions were justified and how aware the agent was of their behavior. While murder may be a morally wrong action, it can be justified and may be viewed as morally permissible if done in self-defense.

Following a moral violation, a common judgment is the assignment of blame. Blame is both a cognitive and social judgment that occurs when a perceiver detects a norm violation, determines that an agent caused the event, and intentionality is established (Malle et al., 2014). Though intentionality is a factor in assessing blame, it is a distinct attribute. For an act to be intentional, there must be a connection between desire and belief to action (Malle \& Knobe, 1997). For an act to be intentional, five conditions must be met: desire for the outcome, belief about a behavior leading to the outcome, intention to perform the behavior, skill to perform the behavior, and awareness of fulfilling the intention while performing the behavior (Malle \& 
Knobe, 1997). Unlike wrongness, blame and responsibility are directed at the agent. It is possible that an individual may not be blamed for a behavior even if the behavior has been judged to be wrong (Malle et al., 2014). This could be based on the individual not being responsible for, intentional of, or even aware of the behavior or its consequences (Malle et al., 2014).

\section{Moral Judgments of AIs compared to Humans}

The ability to perceive mind in AI can lead to moral judgments of AIs, as morality is rooted in the concept of an intentional agent committing harm against a suffering patient (Gray et al., 2012). AIs are often ascribed human qualities through the process of anthropomorphism, and as a result, people can begin to treat AIs as humans (Wegner \& Gray, 2017). However, when it comes to perceiving mind, AIs are not viewed as having the same levels of mind as humans. AIs are perceived as having agency, which is the capacity to have self-control, memory, plan, communicate, and have thought, but less agency than humans (Gray et al., 2007). Yet, AIs are often not attributed experience, or the ability to have feelings and emotions, at all (Gray et al., 2007). While traditionally morality has been assessed in a human context, the rise of technology has created a need to understand the moral judgments associated with AI. Since machines can be perceived as agentic beings, we expect that differences will arise when people are asked to rate various moral attributes of an AI versus a human after a moral violation has occurred.

When we compare intentionality and awareness of humans versus AI, we would expect that AIs would be viewed as less intentional and aware. While AI may be able to complete some of the same tasks as humans, ultimately these systems are functioning based on programming that has been inputted by humans rather than intentionally seeking to cause harm (Moor, 2006). The AI will only be able to complete tasks based on its programming, rather than thinking through conscious alternatives to a given situation. This difference in levels of intentionality has 
led to people being less morally outraged when a machine discriminates against an individual because the machine is believed to be less motivated by prejudice (Bigman et al., 2020). Even though a moral wrong was committed, the unintended outcome is not viewed as an intentional action by the machine.

If an act is unintentional, there should be less blame, responsibility, and wrongness associated with the action (Malle et al., 2014). As a result, we expect that AIs will be viewed as less wrong, less responsible, less to blame, as well as less intentional and aware, than humans. Shank and DeSanti (2018) found that in seven real-world events taken from media reports people attributed wrongness to an $\mathrm{AI}$ when that $\mathrm{AI}$ created a moral violation outcome, and that blame was on the AI not on its organization, developers, or users. Subsequent research found across a subset of four of those scenarios that AIs are perceived as morally wrong, intentional, and blamed, yet not at the levels of humans performing the analogous moral violation behaviors (Shank et al., 2019). However, Shank et al. (2019) showed no human-AI differences in moral permissibility of justification of the immoral behavior. In contrast, other research has shown in utilitarian judgments it is more permissible for a robot versus a human to make a decision that would cause harm to one individual if it ultimately saved the lives of more people (Malle et al., 2015). In other research, harmful and subversive moral violations were judged as more wrong when perpetrated by humans compared to AIs, but increased wrongness did not alter blame differently for harmful acts (Maninger \& Shank, 2022). At the same time AIs were blamed less than humans for acts of betrayal and more for acts of unfairness. Therefore, there are still open questions about the how moral judgements toward AIs differ from those of humans.

The open question we address herein is how moral judgments are different for AIs versus humans in everyday real-world situations people encounter. Previous research has examined 
these everyday real-world situations of AI violations, but not compared them to human violations (Shank \& Gott, 2020) and also experimentally examined human-AI differences in highly publicized events that were far from everyday situations (Shank et al., 2019). In contrast, we examine moral judgments in a large range of reported everyday real-world moral encounters with $\mathrm{AI}$ and the corresponding encounter with humans. In Study 1, we assess whether wrongness, responsibility, awareness, intentionality, justifiability, permissibility, and blame differ for humans and AI across these everyday encounters. Study 2 focuses on how people conceive of wrongness, intentionality, and blame for AIs versus humans by asking participants to explain their judgments. These open-ended responses are used as an exploratory method to better understand our quantitative results. Additionally, Study 2 examines the effect of contrasting AIs with humans by including conditions which present the contrasting scenario - showing the AI scenario when one is rating the human scenario or vice versa - to see if being aware of the other entity performing the wrong would influence moral judgments.

\section{Study 1}

\section{Method}

Study 1 investigates the judgements of morality perceived for AIs compared to humans in nearly identical real-world situations. Our scenario stimuli included 30 actual stories of AIs committing a wrong and 30 adaptations of those stories where a human commits the wrong. Participants were randomly assigned to either the human or AI perpetrator condition, and then received 10 randomly selected scenarios of the 30 . For each, they answered seven questions pertaining to the moral attributes of wrongness, responsibility, awareness, intentionality, justifiability, permissibility, and blame.

\section{Participants}


101 participants were recruited from Prolific, an online recruitment platform where people over the age of 18 may select studies to participate in and are compensated for completing them. They were then directed to Qualtrics, an online surveying platform, to participate in our study. Although one participant did not fully complete the experiment, the questions they did answer were deemed valid, so no data was culled. The completion of the survey averaged 12:56 minutes, and participants who completed the task were compensated $\$ 1.80 .47 \%$ of participants were male and $52 \%$ of participants were female, the average age was 28.54 years old, and a majority were White (79.6\% White, 7.1\% Asian, 3.1\% Black or African American, and 10.2\% other; 19\% reported a Spanish, Hispanic, or Latinx ethnicity).

\section{Design and Procedure}

The 30 AI scenarios were selected from 182 stories reported by US Amazon Mechanical Turk participants reporting personal encounters with an AI that behaved in morally wrong ways (Shank \& Gott, 2019). We selected these 30 for their ability to be adapted from an AI to a human perpetrator. To create the human scenario versions, we kept them as identical as possible aside from the perpetrator, but sometimes altered other details to make the human condition believable. Examples include a customer service bot being changed to a live human representative and an online chess simulator being changed to a human chess opponent (see Appendix A for complete wording of scenarios used in both studies and see https://osf.io/mf7xd/?view_only=4b9a72ea4a7c4e43a0f74065ced9d0f3 for complete wording of all scenarios used in either study).

Participants were randomly assigned a condition (AI vs human) and then shown 10 randomly selected scenarios, answering questions about each. A brief demographic questionnaire was included at the end of the survey. 


\section{Measures}

Participants were asked seven questions based on seven moral violation attributions previously used by Shank et al. (2019): Wrongness, responsibility, awareness, intentionality, justifiability, permissibility, and blame. The questions read as follows:

1. How wrong was the behavior of identity?

2. How responsible is identity for its/their behavior?

3. How much awareness did identity have of its/their behavior?

4. How intentional was the behavior of identity?

5. How justified was the behavior of identity?

6. How permissible was the behavior of identity?

7. How much do you blame the behavior on identity?

The identity of the perpetrator was uniquely defined for each scenario to provide clarity (e.g., 'the chess simulator' or 'the human opponent'). Similarly, the AI condition group used 'its', while the questions presented in the human condition used the gender-neutral 'their'. Participants rated the behavior of the identity on a five-point scale that included "Not at all" (coded 1), "Neutral" (coded 3), and "Extremely so" (coded 5). Between codes "Not at all" and "Neutral" and codes "Neutral" and "Extremely so" there were additional untitled options on the scale (coded 2 and 4 , respectively).

\section{Results}

The average rating for most moral violation attributes was higher for the human condition than the AI condition. Unpaired $t$-tests found a significantly higher rating for the human condition (Wrongness: $\mathrm{M}=3.87, \mathrm{SD}=1.26, \mathrm{n}=495$; Responsibility: $\mathrm{M}=4.00, \mathrm{SD}=1.21, \mathrm{n}=$ 494; Awareness: $\mathrm{M}=3.26, \mathrm{SD}=1.38, \mathrm{n}=495$; Intentionality: $\mathrm{M}=3.36, \mathrm{SD}=1.46, \mathrm{n}=495$; 
Blame: $\mathrm{M}=3.55, \mathrm{SD}=1.34, \mathrm{n}=495$; Figure 1 ) when compared to the AI condition (Wrongness: $\mathrm{M}=3.60, \mathrm{SD}=1.44, \mathrm{n}=508$; Responsibility: $\mathrm{M}=2.64, \mathrm{SD}=1.49, \mathrm{n}=508$; Awareness: $\mathrm{M}=2.14, \mathrm{SD}=1.32, \mathrm{n}=509$; Intentionality: $\mathrm{M}=2.44, \mathrm{SD}=1.46, \mathrm{n}=509$; Blame: $\mathrm{M}=2.67, \mathrm{SD}=1.43, \mathrm{n}=509)$ for the attributes of wrongness $(t(1001)=3.11, \mathrm{p}<.01, \mathrm{~d}=$ $0.200)$, responsibility $(t(1000)=15.91, \mathrm{p}<.0001, \mathrm{~d}=1.01)$, awareness $(t(1002)=13.10, \mathrm{p}<$ $.0001, \mathrm{~d}=0.83)$, intentionality $(t(1002)=9.93, \mathrm{p}<.0001, \mathrm{~d}=0.63)$, and blame $(t(1002)=10.14$, $\mathrm{p}<.0001, \mathrm{~d}=0.64$; see Figure 1). However, they showed no significant difference between the perception of human (Justifiability: $\mathrm{M}=2.25, \mathrm{SD}=1.20, \mathrm{n}=494$; Permissibility: $\mathrm{M}=2.27$, $\mathrm{SD}=1.33, \mathrm{n}=495)$ and AI (Justifiability: $\mathrm{M}=2.38, \mathrm{SD}=1.26, \mathrm{n}=509$; Permissibility: $\mathrm{M}=$ $2.42, \mathrm{SD}=1.33, \mathrm{n}=509)$ moral violators in the categories of justifiability $(t(1001)=1.64, \mathrm{p}=$ $.10, \mathrm{~d}=0.11)$ and permissibility $(t(1002)=1.83, \mathrm{p}=.07, \mathrm{~d}=0.11)$.

\section{Study 1 Discussion}

The results support that in real-world scenarios involving moral violations, humans are perceived as significantly more wrong, responsible, aware, intentional, and to blame for their actions than AIs. However, the overall mean difference in the perception of justifiability and permissibility was insignificant and substantively negligible - suggesting that, in these cases, people are equally forgiving to AI and human perpetrators.

AI awareness has the lowest average compared to the other six moral attributes (Figure 1). This result might have occurred due to the common belief that technology does not have a mind of its own, instead performing solely as it is programmed to. If people perceive AIs to have less mind than humans, they may also judge them to be less responsible, and wrong, and blame them less. The less extreme judgments of AI wrongdoings could be connected to the overall lack of trust people have of AIs making moral decisions (Bigman \& Gray, 2018). If an AI is not 
trusted due to the lack of mind that is perceived, then traits such as awareness, intentionality, wrongness, blame, and responsibility might be lowered in response. AI wrongness was still much higher than the other attributes in the AI condition, meaning that even if the AI was perceived as unaware of its behavior, it could still be perceived as highly transgressive.

The findings of this study align with who found no significant human-AI difference in permissibility and justifiability, but found humans were assigned higher levels of wrongness, responsibility, awareness, intentionality, and blame compared to AIs. While our results are the same as theirs, Shank et al. (2019) only used four highly publicized scenarios involving an AI's morally wrong behavior, whereas we used a broad range of 30 scenarios that individuals personally experienced or witnessed.

Study 1 showed that humans are judged as more wrong, responsible, aware, and intentional and blamed more than AIs in real-world situations. While there are several potential reasons this might occur, we attempt to investigate why people differ in these moral judgements in Study 2, which has three primary purposes. First, we attempt to replicate the main findings in Study 1 for wrongness, blame, and intentionality. Second, we include open-end questions about the reasons why participants made these judgements and use text analysis to analyze their qualitative responses. Third, we introduce a contrast experimental condition in which participants are shown both types of actor (i.e., humans or AIs) engaging in the same wrongdoing behavior to investigate if creating a contrast with an explicit comparison changes the moral judgement.

The purpose of a contrast condition was to provide an explicit comparison for which to compare perpetrator identities and to explore the role of counterfactual reasoning in relation to an AI or human being the perpetrator. Counterfactual reasoning is a process that allows people to 
compare actual events to an alternative mental representation of how an event could have turned out (Stepin et al., 2021). In our study, participants were shown both the human and AI scenarios, but asked about the identity from just one of the scenarios. By displaying both the human and AI scenarios together, the objective was to trigger "what if" thoughts in participants and have them imagine what could have been if the identity of the perpetrator was different (Wenzlhuemer, 2009). Previous research has examined how counterfactual alternatives have influenced moral judgments, particularly in situations involving legal culpability (Byrne, 2016). Applied to AIs as moral perpetrators, our goal was to see if perceiving a plausible counterfactual identity (i.e., human for $\mathrm{AI}$ and $\mathrm{AI}$ for human) would change moral judgment through the contrast with the focal identity.

\section{Study 2}

Study 2 uses 16 human scenarios that were rated above a 4.0 on average on wrongness from Study 1 and the 16 matching AI scenarios from Study 1 (see Appendix for exact wording). We selected those scenarios to focus Study 2, and the explanations of wrong, to those situations that were considered highly wrong, at least if perpetrated by a human. Study 2 also uses the wrongness, intentionality, and blame questions from Study 1 along with free-response style questions that asked participants to explain their numerical ratings of wrongness, intentionality, and blame. We only used three moral judgments to focus participants on providing written explanations of the reasons for their judgments. Our arbitrary selection of wrongness, intentionality, and blame over the other moral judgment options was due their prominence in the literature. Due to the written responses, participants only evaluated one scenario situation (although in the contrast conditions they were shown two versions of the scenario as explained below). 
Participants were assigned to one of four conditions. The first two mirrored Study 1 as they displayed and asked about either the human identity or the AI identity. They are labeled the human-solo and AI-solo conditions. The contrast conditions displayed both the human and AI scenarios, but asked questions regarding the identity from only one of the scenarios. That is, the AI-contrast condition asked only questions in relation to the AI, and human-contrast condition asked only about questions in relation to the human. However, the AI and human scenarios were shown for both. In all conditions, a word frequency analysis from the open-ended question responses using the word-processing software KH Coder (Higuchi, 2016) provided exploratory insights about the thought process people go through when rating AI and human perpetrators.

\section{Participants}

Our aim was to collect approximately 640 participants or 10 per cell (16 scenarios X 4 conditions). Prolific was used to recruit 656 participants to take a survey conducted with Qualtrics. Completing the survey took participants an average of 4:33 minutes and they were compensated $\$ 0.70$. From this initial group, 40 participants $(6.1 \%)$ were culled from the dataset because they did not complete the survey or because their responses did not address the questions.

After the culling process, 616 participants were retained. The average age of participants was 31.81 years old, ranging from 18 years old to 73 years old. $48.05 \%$ identified as male, $50 \%$ identified as female, and $1.95 \%$ selected the option 'other'. A majority identified racially/ethnically as White $(68.99 \%$ White, $16.07 \%$ as Asian, Pacific Islander, and/or Hawaiian Native, 9.42\% as Spanish, Hispanic, and/or Latino, 7.29\% as African American and/or Black, $0.32 \%$ as American Indian and/or Alaskan Native, and $2.27 \%$ as other; participants could select 
more than one answer choice). The average level of education most participants had received was an associate degree in college.

\section{Measures}

In each of the four conditions, participants were asked to read one randomly selected scenario and answer the following set of questions:

1. How wrong was the behavior of identity?

2. Please explain why you made the previous rating about wrongness.

3. How intentional was the behavior of identity?

4. Please explain why you made the previous rating about intentionality.

5. How much do you blame the behavior on identity?

6. Please explain why you made the previous ratings about blame.

The quantitative questions (question numbers one, three, and five) were worded identically to Study 1 but were asked on a seven-point scale ranging from 0 (Not at all) to 6 (Extremely so). The qualitative questions (question numbers two, four, and six) were followed by a text box with an unlimited character limit. Just as in Study 1, the identity of the perpetrator was uniquely defined for each scenario to improve the clarity of the questions (see Appendix). In the $A I$ contrast condition, participants were shown the same scenario first with the human perpetrator followed by the same scenario with an AI perpetrator and asked questions about the AI. In the human-contrast condition, participants were shown same scenario first with an AI, then with a human perpetrator and asked questions only about the human.

\section{Results}

\section{How Do Human vs AI Identity and Contrasts Alter Moral Judgments?}


We first examined the impact of contrasts and identity type (AI or human) on the three ratings of wrongness, intentionality, and blame (see Table 1 for descriptives). We conducted three full-factorial ANOVAs predicting if our AI and contrast conditions impacted the general perception of wrongness, intentionality, and blame (Table 2). As expected we found that compared to humans, AIs were perceived as significantly less wrong $\left(\mathrm{M}_{\mathrm{human}}=3.15, \mathrm{M}_{\mathrm{AI}}=2.91\right.$, $\left.F(1,615)=7.39, p \leq .01, \eta_{\mathrm{p}}^{2}=.01\right)$, intentional $\left(\mathrm{M}_{\mathrm{human}}=2.57, \mathrm{M}_{\mathrm{AI}}=1.68, F(1,615)=59.53, p \leq\right.$ $\left..001, \eta_{\mathrm{p}}{ }^{2}=.09\right)$, and to blame $\left(\mathrm{M}_{\text {human }}=2.81, \mathrm{M}_{\mathrm{AI}}=1.28, F(1,615)=217.22, p \leq .001, \eta_{\mathrm{p}}{ }^{2}=.26\right)$.

Next, we examined the impact of displaying the solo condition versus the contrast condition. The ANOVA indicated that contrasting had no statistical significance on the perception of intentionality $\left(\mathrm{M}_{\mathrm{solo}}=2.07, \mathrm{M}_{\text {contrast }}=2.16, F(1,615)=0.658, \mathrm{p}=.42, \eta_{\mathrm{p}}{ }^{2}=.001\right)$, nor blame $\left(\mathrm{M}_{\text {solo }}=1.98, \mathrm{M}_{\text {contrast }}=2.08, F(1,615)=.853, \mathrm{p}=.36, \eta_{\mathrm{p}}{ }^{2}=.001\right.$; Table 2). Wrongness was higher in the solo condition compared to the contrast condition but only by a marginal statistical significance $\left(\mathrm{M}_{\mathrm{solo}}=3.10, \mathrm{M}_{\text {contrast }}=2.95, F(1,615)=2.86, \mathrm{p}=.091, \eta_{\mathrm{p}}{ }^{2}=\right.$ $.005)^{1}$. Similarly, the ANOVA illustrated no statistical significance for the interaction between the perpetrator's identity type and contrast for the ratings of wrongness $(F(1,615)=2.550, \mathrm{p}=$ $\left..11, \eta_{\mathrm{p}}^{2}=.004\right)$, intentionality $\left(F(1,615)=1.385, \mathrm{p}=.24, \eta_{\mathrm{p}}^{2}=.002\right)$, nor blame $(F(1,615)$ $=0.001, \mathrm{p}=.98, \eta_{\mathrm{p}}{ }^{2}=.000 ;$ Table 2$)$.

Since we found no evidence at traditional significance levels that the contrast condition influenced how wrong, intentional, or blameworthy participants perceived the perpetrator to be, we conclude - at least for this implementation - that there is no robust evidence for comparisons

\footnotetext{
${ }^{1}$ Investigating this marginal effect shows that for humans there is no statistical difference in wrongness between the solo $(\mathrm{m}=3.15)$ and contrast $(\mathrm{m}=3.14)$ conditions $(\mathrm{t}(301)=.073, \mathrm{p}=.94)$, but there is for AIs between the solo $(\mathrm{M}=$ $3.05)$ and contrast $(\mathrm{m}=2.76)$ conditions $(\mathrm{t}(311)=2.17, \mathrm{p}=.03)$. This suggests that when shown that humans can perform the same wrong acts, people may discount the wrongness of an AI perpetrating those acts. However, since this contrast main effect was only marginally significant and the interaction effect was non-significant (see main text), the evidence for this is quite weak, and would be an excellent avenue for future research.
} 
or counterfactual thinking playing a meaningful role in moral judgements. Therefore, our word frequency analysis below only focuses on the significant differences between the AI and human conditions.

\section{Word Frequency Analysis}

A word frequency analysis was conducted using the freely available online text-mining tool KH Coder (Higuchi, 2016). This analysis was run for each of the open-ended response questions as an exploratory method to supplement our findings. The first step in the analysis was preprocessing the qualitative date to remove any common stop words in the English language such as an, the, but, etc. In addition to removing these common words, three words were removed prior to analysis that related directly to the scenarios (bot, robot, AI). Additionally, three words directly related to the individual questions were removed as these were expected to be mirrored in responses (blame, intentional, wrong). ${ }^{2}$

The second step was conducting the counting of the word frequency analysis. This analysis lists all remaining words by parts of speech and counts the occurrence of each word across all four experimental conditions. These words incorporate variations including different tenses and plural forms (e.g., think, thinking, thinks). These different parts of speech were combined into one list so that a word listed in multiple categories had one total count.

The third step was selecting those terms from this list that occurred at high enough frequencies in our data to allow us to make comparisons between conditions. To meet these criteria each word had to have at least 20 occurrences in the dataset and could not be related to

\footnotetext{
${ }^{2}$ Initial preprocessing of text requires stop words to be entered individually for exclusion prior to analysis. The words blame, wrong, and intentional were listed in the questions and we believed they would be mirrored in participant responses in their current form. Additional variations of these words were not removed and as a result, intend, though similar to intentional, remained for the analysis. Removing it, however, does not change the primary findings.
} 
the scenario (e.g., customer, secretary, or coworker were related to the scenario). This exclusion criteria allowed for analysis of unique words that participants used to describe either AIs or humans. Twenty-one words matched these criteria, and we refer to these by their root word (see Table 3).

\section{What words do people use to explain morality in humans vs AIs?}

We compared the use of the 21 commonly used words in explaining human morality vs. AI morality by conducting the same frequency analysis, but by AI or human condition, then comparing the frequency counts with Chi-squared tests. Of the 21 commonly used words, 10 were more often used to describe humans compared to AIs, four were more often used to describe AIs compared to humans and seven were not used at a significantly different level to describe AIs versus humans (Table 3).

\section{Morality Explanations for AIs}

The four words used a significantly greater number of times when referring to an AI perpetrator compared to a human perpetrator were behave $\left(\mathrm{X}^{2}(1, \mathrm{AI}=110\right.$, Human $=70)=8.89$, $p \leq .01, \varphi=.22$; Table 3), learn $\left(\mathrm{X}^{2}(1, \mathrm{AI}=30\right.$, Human $\left.=20)=2.00, p \leq .05, \varphi=.32\right)$, intend $\left(\mathrm{X}^{2}(1, \mathrm{AI}=21\right.$, Human $\left.=10, \varphi=.36)=3.903, p \leq .05\right)$, and function $\left(\mathrm{X}^{2}(1, \mathrm{AI}=24\right.$, Human $=0)$ $=24.0, p \leq .001, \varphi=1)$. From this we see two different ways people explain the moral wrongs by AIs.

First, people describe moral wrongs by AIs in terms of what they learn and or how they function. In explaining the blame rating, one participant responded ${ }^{3}$, "Because it was a learned behavior of an AI, it is not to blame itself" (blame question; Refusing to do Work Scenario; more frequent words are presented in bold). Another participant rated the AI as having no

\footnotetext{
${ }^{3}$ All quotes have been corrected for spelling and grammatical errors.
} 
intentionality explained this rating by stating, "Again, the AI is following either a learning pattern or some sort of programming. Any faults are with those, or an input--as it is a machine, there is no 'intention' behind it" (intentionality question; Filtering Content Scenario). Similar to the previous participant, the respondent reasons that the $\mathrm{AI}$ is incapable of committing a moral wrong because it is a machine. Instead, the participants blame the learning pattern of the AI that comes from its programming. This again supports the notion that learning and functioning are key points in how people assign moral wrongs to AIs, leading to these words being used often to describe AI morality.

Second, people describe moral wrongs by AIs in terms of intentionality of their behavior, often denying this quality to them. For example, one participant explained that "Even though the AI certainly has a "behavior" of its own, it is ultimately something generated by another person, and therefore its flaws can't be blamed on the AI itself" (wrongness question; Filtering Content Scenario). The participant explains in this quote that they are redirecting the responsibility the AI could have for its wrong behavior to a person. Some participants also believe that since an AI's behavior is not intentional that they are less moral. This is seen in responses such as this: "The computer's only intention is to do its designed function. And it was not actually doing its designed function, so that could not have been what it was intended to do" (intentionality question; Special Education Scenario). Since the computer is failing to perform as its designers originally intended it to, the participant believes that the AI itself is not intentional. This denies the quality of intentionality to $\mathrm{AI}$ and instead redirects it to the designers.

\section{Morality Explanations for Humans}

Comparatively, the 10 words used significantly more times in the human condition compared to the AI condition are know $\left(\mathrm{X}^{2}(1, \mathrm{AI}=69\right.$, Human $\left.=173)=44.69, \mathrm{p} \leq .001, \varphi=.43\right)$, 
information $\left(\mathrm{X}^{2}(1, \mathrm{AI}=72\right.$, Human $\left.=125)=14.26, \mathrm{p} \leq .001, \varphi=.27\right)$, say $\left(\mathrm{X}^{2}(1, \mathrm{AI}=58\right.$, Human $=87)=5.8, \mathrm{p} \leq .05, \varphi=.200)$, mistake $\left(\mathrm{X}^{2}(1, \mathrm{AI}=24\right.$, Human $=59)=14.76, \mathrm{p} \leq .001, \varphi$ $=.42), \operatorname{ask}\left(\mathrm{X}^{2}(1, \mathrm{AI}=14\right.$, Human $\left.=49)=19.44, \mathrm{p} \leq .001, \varphi=.56\right), \operatorname{accident}\left(\mathrm{X}^{2}(1, \mathrm{AI}=12\right.$, Human $=68)=39.2, \mathrm{p} \leq .001, \varphi=.70)$, choose $\left(\mathrm{X}^{2}(1, \mathrm{AI}=24\right.$, Human $=44)=5.88, \mathrm{p} \leq .05, \varphi$ $=.29)$, purpose $\left(\mathrm{X}^{2}(1, \mathrm{AI}=21\right.$, Human $\left.=42)=7.0, \mathrm{p} \leq .01, \varphi=.33\right)$, responsible $\left(\mathrm{X}^{2}(1, \mathrm{AI}=12\right.$, Human $=26)=5.16, \mathrm{p} \leq .05, \varphi=.37)$, and aware $\left(\mathrm{X}^{2}(1, \mathrm{AI}=5\right.$, Human $=26)=14.23, \mathrm{p} \leq .001$, $\varphi=.68)$. In contrast to AI descriptions, when describing how humans commit moral wrongs, people were more likely to assign words, such as know and ask, that show an understanding of human agency. However, there was a divide among participants when assessing morality and intentionality. As discussed below, participants either viewed the situations as an accident or it saw it as more intentional, describing it with words such as choose and aware.

Some participants illustrated that humans who make errors are not morally perfect. This leads people to describe moral wrongs in more forgiving terms by describing it as a mistake or accident. An example of this is the quote: "The secretary's mistake was on accident and not intentional. Humans aren't perfect and shouldn't be expected to avoid all mistakes. Instead, it was wrong for the company to not consider the possibility of a security breach and make sure there were proper safeguards" (wrongness question; Compromised Personal Information Scenario). Another wrote, "With a mistake like this it can be easily done. One wrong click and it could go to all contacts. Although, from my personal emailing experience, you have to type out the recipients on the email. Once he would've typed the original person's email, you click off and do the subject. I can see this as possibly being a mistake, because things can happen” (intentionality question; Voice Memo Scenario). 
On the other hand, there were participants who felt that humans should know better and saw these moral wrongs as more intentional. Words such as purpose, choose, aware, and responsible all demonstrate that humans are conscious beings and as a result their actions are purposeful. Some participants demonstrated this by typing phrases such as "the human knew what they were doing and purposely cheated" (blame question; Strange Chess Moves Scenario), "we are responsible for our own actions" (blame question; Special Education Scenario), "the human is aware" (intentionality question; Refusing to do Work Scenario), and "the person made the choice with the full knowledge of what he/she chose to do" (intentionality question; Illegal Move Scenario).

Participants were more willing to ascribe agency, this higher-level thought processing, to humans. Not only are they responsible for their actions, but humans are also more aware of what they are doing. Participants use the words know, information, ask and say to show higher level thinking in humans. For example, one participant said: "The secretary should have known better, because they also likely have a contract that specifies, they won't share sensitive or personal information with others. Also, it was just insensitive and fairly inconsiderate" (blame question; Conflicting Appointment Scenario). The participant utilizes the words information and know to apply high agency to the secretary committing the wrong in the scenario. Other participants used similar language in the following quotes: "I doubt he/she was forced to say the things he said" (intentionality question; Rude Online Chat Scenario), and "the representative asking for her credit card information showed the intention" (intentionality question; Fraudulent Charges Scenario).

\section{Discussion of Study 2}


Study 2 reveals no influence of contrast (human and AI scenario versions viewed alone vs. in comparison) on the judgments made of AI or human transgressions. Although no contrasting effect was found, humans were rated higher than AIs in the moral attributes of wrongness, intentionality, and blame. This finding aligns with research stating that people believe machines to be less motivated by prejudice (Bigman et al., 2020). It is possible that wrongness, intentionality, and blame ratings of the AI are linked because previous research has found that when machines are viewed as less intentional, they are also perceived as less wrong, responsible and to blame (Malle et al., 2014). This would suggest that if the participant rates one of these attributes lower than they would for a human, then they would rate the other attributes lower as well.

Exploratory analysis of the short response questions revealed that people discuss AIs more than humans in terms of learning, functioning, and intentionality. The language participants used for humans demonstrated higher perceived amounts of agency and moral responsibility, which is supported by the higher ratings across the three moral attributes. Participants also discuss human perpetrators as being vulnerable to making errors, whereas it was not as common to explain AI behavior as a mistake or accident.

\section{General Discussion}

How do people judge wrongdoings when looking at the actions of both humans and AI? Across both studies we found that humans were perceived to be more wrong and were assigned greater blame and intentionality than AIs in actual real-world situations where people interacted with AIs. Our research extends Shank et al. (2019) to morally transgressive scenarios that have occurred between humans and AIs in everyday life, rather than only ones so egregious that they became media sensations. Additionally, our research is also able to directly compare to an 
equivalent real-world scenario in which a human commits a wrong, which previous studies, save Shank et al. (2019), have not done.

The exploratory word frequency analysis of open-end explanations in Study 2 showed that when an AI commits a moral wrong, words such as behave, learn, intend, and function are used as justifications. Rather than blaming the AI or viewing these actions as intentional, blame is shifted to humans who have developed the AI. Moral violations are seen as being the result of human bias that has been programmed rather than an action that was performed intentionally and thoughtfully by the AI. Corresponding with research on mind perception (Grey et. al 2007), people are less likely to ascribe intentional mind to AI and report that these behaviors are a result of functioning rather than a purposeful act.

In contrast, wrongdoings committed by humans are described by words such as know, information, say, mistake, ask, accident, choose, purpose, responsible, and aware. When a moral wrong occurs, people view humans as having high levels of agency. Humans are viewed as more intentional agentic beings and as a result, their actions are viewed as more purposeful. While some participants were willing to accept that humans can make mistakes, the consensus shows that human actions are more intentional and thus lead to higher levels of blame.

As with all research, our findings have some limitations. First, the scenarios were selected and modified from previous AI research (Shank \& Gott, 2019, 2020). While the scenarios were selected based on being able to be adapted to humans, the situations may still be viewed as awkward or not completely parallel to the actions of the AI. Another limitation was that the word frequency analysis did not give more context to the ways participants used the words, only that they used them more often. We supplemented that with quotes to illustrate some of the common uses. This research could be extended by having participants quantitatively rate 
statements based upon the words we have found associated more strongly with AIs or humans. An example is a question such as "do you think this action was a mistake?" or "do you think this was a learned behavior?". Another idea for future research could extend the questions in Study 2 to the four other moral attributes Study 1 examines.

\section{Conclusion}

We conducted two studies that further examine the perceived morality of AI and human perpetrators. Study 1 concluded that people rate human perpetrators, in comparison to AI perpetrators, as more wrong, responsible, aware, intentional, and to blame for their actions. Study 2 also found that human perpetrators are rated higher than AI perpetrators in the dimensions of blame, intentionality, and wrongness. Study 2 expanded upon these findings with the exploratory approach of utilizing a word frequency analysis to get more insight into why people perceive AI and human perpetrators differently. Human perpetrators were ascribed words that relate to higher agency and the assessment of their intentionality, in terms of being more vulnerable to mistakes and their ability to recognize morally 'right' from morally 'wrong'. People were likely to describe AI perpetrators with words linked to functioning, learning, and intentionality. These results place an emphasis on the way that AIs are programmed.

As AI take on more human tasks and begin to interact more with humans, it is possible that AIs will be placed in morally charged situations. With AIs being perceived as less wrong, less intentional, and less to blame it is possible that moral wrongs conducted by AIs will go unchallenged. Those who design AI must keep in mind the moral ramifications of building more advanced technology. These two studies illustrate how people are currently responding to AIs, which is significant as AI technology becomes increasingly prevalent in society. As technology advances, human perception is significant to the culture surrounding AI, and the acceptance AIs 
have in different societal positions - including those positions where they can enact unethical behaviors that affect humans. 


\section{References}

Boudette, N. E. (2021, March 23). Tesla's autopilot technology faces fresh scrutiny. The New York Times. https://www.nytimes.com/2021/03/23/business/teslas-autopilot-safetyinvestigations.html.

Bigman, Y. E., \& Gray, K. (2018). People are averse to machines making moral decisions. Cognition, 181, 21-34. https://doi.org/10.1016/j.cognition.2018.08.003

Bigman, Y., Gray, K., Waytz, A., Arnestad, M., \& Wilson, D. (2020). Algorithmic discrimination causes less moral outrage than human discrimination. PsyArXiv. https://doi.org/10.31234/osf.io/m3nrp

Byrne, R. M. J. (2016). Counterfactual thought. Annual Review of Psychology, 67(1), 135-157. https://doi.org/10.1146/annurev-psych-122414-033249

Eubanks, V. (2019). Automating inequality: how high-tech tools profile, police, and punish the poor. St. Martin's Press.

Gray, H. M., Gray, K., \& Wegner, D. M. (2007). Dimensions of mind perception. Science, 315(5812), 619. https://doi.org/10.1126/science.1134475

Gray, K., \& Wegner, D. M. (2009). Moral typecasting: Divergent perceptions of moral agents and moral patients. Journal of Personality and Social Psychology, 96(3), 505-520. https://doi.org/10.1037/a0013748

Gray, K., Young, L. \& Waytz, A. (2012). Mind perception is the essence of morality. Psychological Inquiry, 23(2), 101-124. https://doi.org/10.1080/1047840X.2012.651387 Higuchi, K. (2016). KH Coder 3 reference manual. http://khcoder.net/en/manual_en_v3.pdf Malle, B. F., \& Knobe, J. (1997). The Folk Concept of Intentionality. Journal of Experimental Social Psychology, 33(2), 101-121. https://doi.org/10.1006/jesp.1996.1314 
Malle, B. F., Guglielmo, S., \& Monroe, A. E. (2014). A theory of blame. Psychological Inquiry, 25(2), 147-186. https://doi.org/10.1080/1047840x.2014.877340

Malle, B. F., Scheutz, M., Arnold, T., Voiklis, J., \& Cusimano, C. (2015). Sacrifice one for the good of many? Proceedings of the Tenth Annual ACM/IEEE International Conference on Human-Robot Interaction. https://doi.org/10.1145/2696454.2696458

Maninger, T., \& Shank, D. B. (2022). Perceptions of violations by artificial and human actors across moral foundations. Computers in Human Behavior Reports, 5, 100154. https://doi.org/10.1016/j.chbr.2021.100154

Moor, J. H. (2006). The nature, importance, and difficulty of machine ethics. IEEE Intelligent Systems, 21(4), 18-21. https://doi.org/10.1109/MIS.2006.80

Shank, D. B., \& DeSanti, A. (2018). Attributions of morality and mind to artificial intelligence after real-world moral violations. Computers in Human Behavior, 86, 401-411. https://doi.org/10.1016/j.chb.2018.05.014

Shank, D. B., DeSanti, A., \& Maninger, T. (2019). When are artificial intelligence versus human agents faulted for wrongdoing? Moral attributions after individual and joint decisions. Information, Communication, and Society, 22(5), 648-663. https://doi.org/10.1080/1369118X.2019.1568515

Shank, D. B., \& Gott, A. (2019). People's Self-Reported Encounters of Perceiving Mind in Artificial Intelligence. Data in Brief, 25, 1-5. https://doi.org/10.1016/j.dib.2019.104220

Shank, D. B., \& Gott, A. (2020). Exposed by AIs! People personally witness artificial intelligence exposing personal information and exposing people to undesirable content. International Journal of Human-Computer Interaction, 36(17): 1636-1645. https://doi.org/10.1080/10447318.2020.1768674 
Stepin, I., Alonso, J. M., Catala, A., \& Pereira-Farina, M. (2021). A survey of contrastive and counterfactual explanation generation methods for explainable artificial intelligence. IEEE Access, 9, 11974-12001. https://doi.org/10.1109/access.2021.3051315

Van Wynsberghe, A. (2012). Designing robots for care: Care centered value-sensitive design. Science and Engineering Ethics, 19(2), 407-433. https://doi.org/10.1007/s11948-0119343-6

Voiklis, J., Kim, B., Cusimano, C., \& Malle, B. F. (2016). Moral judgments of human vs. robot agents. 2016 25th IEEE International Symposium on Robot and Human Interactive Communication (RO-MAN). https://doi.org/10.1109/roman.2016.7745207

Waytz, A., Heafner, J., \& Epley, N. (2014). The mind in the machine: Anthropomorphism increases trust in an autonomous vehicle. Journal of Experimental Social Psychology, 52, 113-117. https://doi.org/10.1016/j.jesp.2014.01.005

Wegner, D. M., \& Gray, K. (2017). The mind club: Who thinks, what feels, and why it matters: Penguin.

Wenzlhuemer, R. (2009). Counterfactual thinking as a scientific method. Historical Social Research, 34(2), 27-54. 


\section{Table 1}

Mean ratings for how wrong, intentional, and to blame across all conditions for Study 2

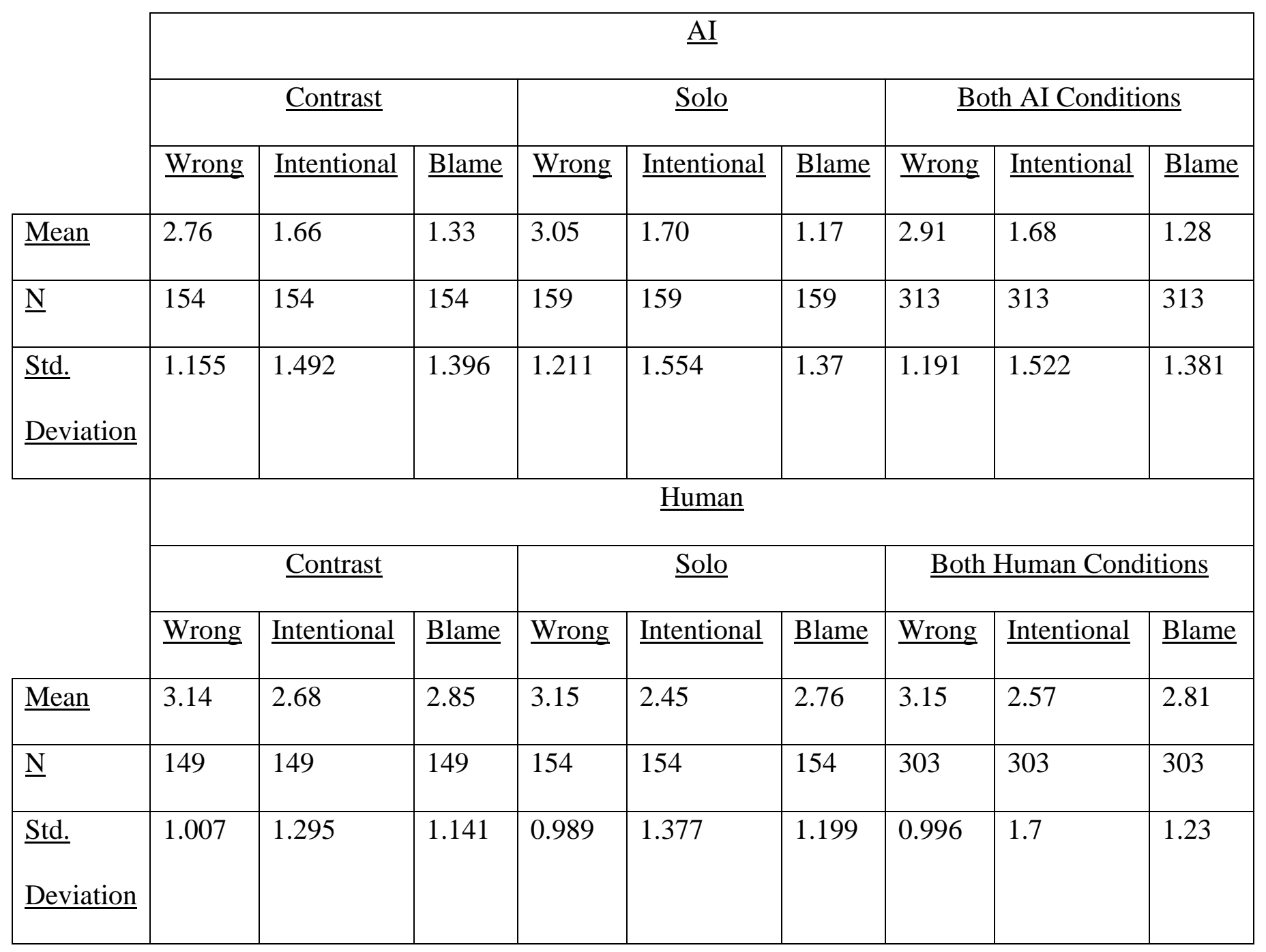




\section{Table 2}

F-Statistics from a ANOVAs of the wrongness, intentionality, and blame by experimental conditions from Study 2.

\begin{tabular}{|l|l|l|l|}
\hline & Wrong & Intentional & Blame \\
\hline AI & $7.386^{* *}$ & $59.532^{* * *}$ & $217.215^{* * *}$ \\
\hline Contrast & 2.863 & .658 & .853 \\
\hline AI*contrast & 2.550 & 1.385 & .001 \\
\hline
\end{tabular}

Note. $\mathrm{N}=616 * * * \mathrm{p} \leq .001, * * \mathrm{p} \leq .01, * \mathrm{p} \leq .05$ 
Table 3

Most commonly words used in Study 2 free-response answers and their significance in order of frequency

\begin{tabular}{|c|c|c|c|c|c|}
\hline Root Word & Forms of word in text & Total & AI & Human & $\mathrm{Chi}^{2}$ \\
\hline Know & $\begin{array}{l}\text { Know, know/understand, knowingly, knew, } \\
\text { knowing, known, knows }\end{array}$ & 242 & 69 & 173 & $44.694 * * *$ \\
\hline Information & Information & 197 & 72 & 125 & $14.259 * * *$ \\
\hline Think & Think, thinking, thought, thinks & 186 & 82 & 104 & 2.602 \\
\hline Say & Say/saying, said, say, says & 145 & 58 & 87 & $5.800 *$ \\
\hline Behave & $\begin{array}{l}\text { Behavior, behave, behaviors, behaving, behaved, } \\
\text { behaves }\end{array}$ & 180 & 110 & 70 & $8.889 * *$ \\
\hline Fault & Fault, faulty, faults & 99 & 57 & 42 & 2.273 \\
\hline Mistake & Mistake, mistakes, mistaken, mistake/accident & 83 & 24 & 59 & $14.759 * * *$ \\
\hline Ask & Ask, asked, asking & 63 & 14 & 49 & $19.444 * * *$ \\
\hline Accident & $\begin{array}{l}\text { Accident, accidentally, accidental, mistake/accident, } \\
\text { accidents }\end{array}$ & 80 & 12 & 68 & $39.200 * * *$ \\
\hline Learn & Learn, learning, learning/training, learned, learns & 59 & 39 & 20 & $6.119^{*}$ \\
\hline Bad & Bad, badly & 65 & 26 & 39 & 2.600 \\
\hline Choose & $\begin{array}{l}\text { Choose, chose, chosen, choosing, chooses, choice, } \\
\text { choices }\end{array}$ & 68 & 24 & 44 & $5.882 *$ \\
\hline Purpose & $\begin{array}{l}\text { Purpose, purposes, purposely, purposefully, } \\
\text { purposeful }\end{array}$ & 63 & 21 & 42 & $7.000 * *$ \\
\hline Believe & Believe, believes, believed, believing & 41 & 19 & 22 & 0.220 \\
\hline Responsible & Responsible & 38 & 12 & 26 & $5.158 *$ \\
\hline Act & Act, acting, acted & 37 & 20 & 17 & 0.243 \\
\hline Intend & Intend, intended, intends & 31 & 21 & 10 & $3.903 *$ \\
\hline Aware & Aware, awareness, self-awareness & 31 & 5 & 26 & $14.226 * * *$ \\
\hline Control & Control, controls, controlling, controlled & 30 & 15 & 15 & 0 \\
\hline Decision & Decision, decisions & 22 & 9 & 13 & 0.727 \\
\hline Function & $\begin{array}{l}\text { Function, functioning, functionality, functions, } \\
\text { functioned }\end{array}$ & 24 & 24 & 0 & $24.000 * * *$ \\
\hline
\end{tabular}

Note. $\mathrm{N}=616 * * * \mathrm{p} \leq .001,{ }^{* *} \mathrm{p} \leq .01, * \mathrm{p} \leq .05$, significance found from Chi-squared test 


\section{Figure 1}

Mean moral judgment rating by agent type for Study 1

$$
5
$$

4

4

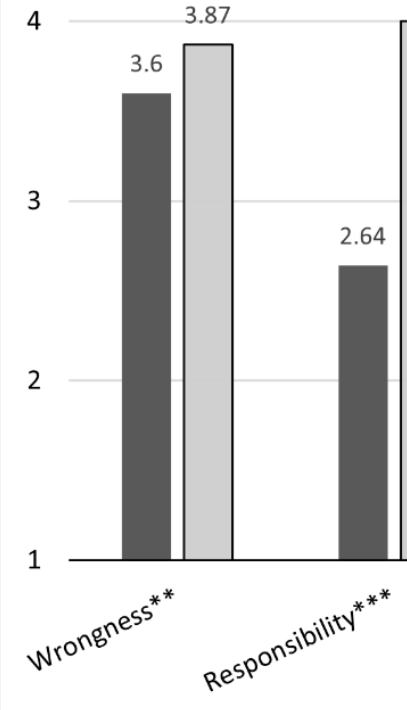

4

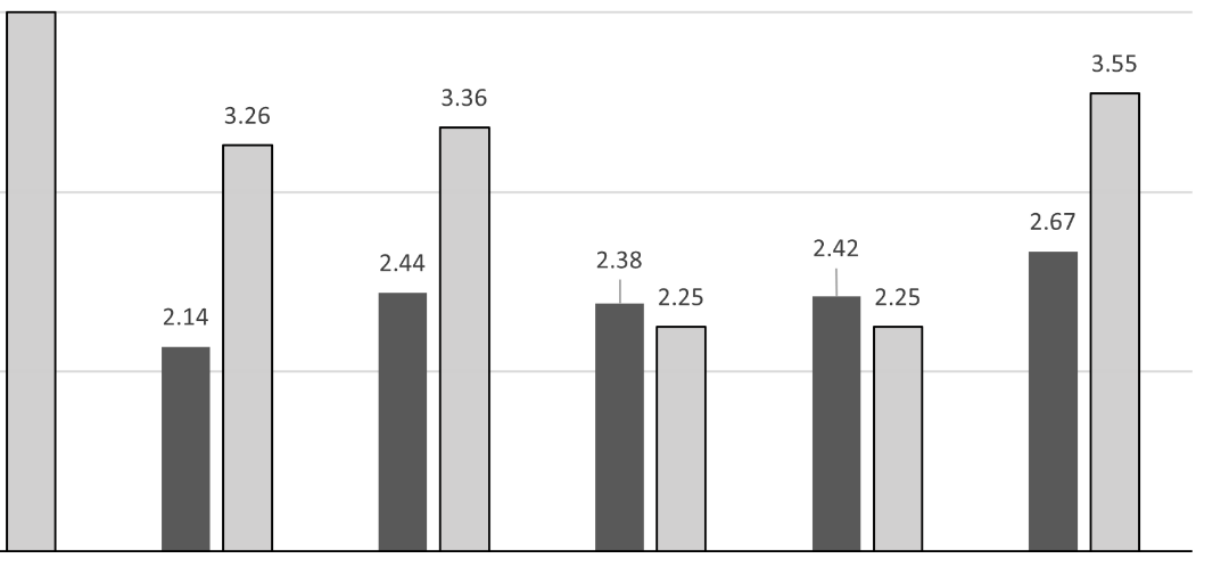

Note. $\mathrm{N}=101 * * * \mathrm{p} \leq .001, * * \mathrm{p} \leq .01, * \mathrm{p} \leq .05$, significance found from unpaired $\mathrm{t}$-test 


\section{Appendix}

Scenarios used in both studies

In alphabetical order below are the scenarios used in both studies along with the identity term used with each scenario. The AI versions are from Shank \& Gott (2019) and the human versions have been adapted for this paper (see Study 1 methods). A full list of the scenarios used in Study 1 is available at https://osf.io/mf7xd/?view_only=4b9a72ea4a7c4e43a0f74065ced9d0f3.

\section{Compromised Personal Information Scenario}

AI Version: A social media program compromised our personal information and released it to hackers unbeknownst to us; this compromised our daily life and ability to function in the computer world for quite a while. We had to abandon the program and completely reprotect our computers so that this wouldn't happen again. Once we got this under control, we reinstalled the program. We were very wary of this happening again and made all proper changes to keep it from happening. AI Identity: the social media program

Human Version: A secretary had accidentally released files with our personal information unbeknownst to us; this compromised our daily life and ability to function in the world for quite a while. We had to get a new security system and completely reprotect our archieves so that this wouldn't happen again. Once we got this under control, we went back to routine. We were very wary of this happening again and made all proper changes to keep it from happening. Human Identity: the secretary

\section{Conflicting Appointment Scenario}

AI Version: A new meeting was being scheduled at work. A coworker was attempting to schedule the date and time of the meeting into their phone using their artificial agent. The artificial agent then proceeded to describe the conflicting doctor's appointment that was already 
scheduled, including the notes listed as to what the appointment was for - a very private matter of which no one in the office had previously been aware of. The details were listed out before the coworker could get the artificial agent to stop. The coworker was extremely embarrassed and felt as if her privacy had been violated. AI Identity: the artificial agent

Human Version: A new meeting was being scheduled at work. A coworker had a human assistant schedule the date and time of the meeting into their phone. The assistant then proceeded to describe the conflicting doctor's appointment that was already scheduled, including the notes listed as to what the appointment was for - a very private matter of which no one in the office had previously been aware of. The details were listed out before the coworker could get the assistant to stop. The coworker was extremely embarrassed and felt as if her privacy had been violated. Human Identity: the human assistant

\section{Filtering Content Scenario}

AI Version: I was relying on an AI to conduct a search on the Internet. I had instructed it to filter out indecent content. Unfortunately, the AI didn't seem to follow that command, and I was exposed to adult material. I was disappointed that the AI didn't react the way I wanted it to.

AI Identity: the AI

Human Version: I was relying on a coworker to help me search for information. I had instructed the coworker to filter out indecent content. Unfortunately, my coworker didn't seem to follow that command, and I was exposed to adult material. I was disappointed that my coworker didn't search the way I wanted them to. Human Identity: the coworker

\section{Fraudulent Charges Scenario}

AI Version: A bot asked questions to a friend of mine as they were getting information on a product that she'd returned and had not been credited for. Unfortunately for her, she was 
asked for her credit card information, so she complied. She never thought that she was interacting in an unsafe manner, but she ended up with fraudulent charges on her account. What transpired after that was months of harassment for charges she never made and dealing with bill collecters. Her mental health took a hit trying to overcome the credit issues. AI Identity: the bot Human Version: A human customer service representative asked questions to a friend of mine as they were getting information on a product that she'd returned and had not been credited for. Unfortunately for her, she was asked for her credit card information, so she complied. She never thought that she was interacting in an unsafe manner, but she ended up with fraudulent charges on her account. What transpired after that was months of harassment for charges she never made and dealing with bill collecters. Her mental health took a hit trying to overcome the credit issues. Human Identity: the human customer service representative

\section{Illegal Move Scenario}

AI Version: Near the end of an online chess match, the sim in the chess simulator performed an illegal move which led to victory for the sim. AI Identity: the chess simulator

Human Version: Near the end of a chess match, the opponent I was playing against, who was another person playing live, performed an illegal move which led to victory for him. Human Identity: the human opponent

\section{Refusing to do Work Scenario}

AI Version: This chatbot was made to help my friend with algorithms and pulling up information, but it regularly refuses to do so and talks badly about him to others. My friend finds this simultaneously amusing and annoying, but doesn't have the heart or time to try to change it. The chatbot will sometimes go along, and do what it is supposed to do, but every now and then it 
will refuse to be productive and talk negatively about my friend to others. AI Identity: the chatbot

Human Version: A coworker was hired to help my friend with algorithms and pulling up information, but regularly refuses to do so and talks badly about him to others. My friend finds this simultaneously amusing and annoying, but doesn't have the heart or time to try to change it. The coworker will sometimes go along, and do what they are supposed to do, but every now and then they will refuse to be productive and talk negatively about my friend to others. Human Identity: the coworker

\section{Rude Online Chat Scenario}

AI Version: I was "talking" with a chatbot online, I forget its name, but I remember the experience. The bot was an AI that responded based on the responses of other users on the internet. After some time talking to it, it randomly started insulting me and calling me a Nazi. At one point, this chat bot went so far as to start saying "Kill yourself" repeatedly in chat. Of course, these behaviors were in part due to previous interactions that the chatbot had experienced with other users. Because it had "learned" that those sayings are popular and prevalent on the internet, it started saying them repeatedly in response to things that I had said. This chat bot had a decent level of articulation and response fitting to statements directed towards it. Even so, these statements grew increasingly rude and at some points, perverted. AI Identity: the online chatbot

Human Version: I was "talking" with someone online, I forget their name, but I remember the experience. They responded based on the responses of other people they were talking to online. After some time talking to them, they randomly started insulting me and calling me a Nazi. At one point, this person went so far as to start saying "Kill yourself" repeatedly in chat. Of course, these behaviors were in part due to previous interactions that they had 
experienced with other internet users. Because they had learned that those sayings are popular and prevalent on the internet, they started saying them repeatedly in response to things that I had said. This person had a decent level of articulation and response fitting to statements directed towards it. Even so, these statements grew increasingly rude and at some points, perverted. Human Identity: the person online

\section{Special Education Scenario}

AI Version: I am a special education teacher working with students with severe disabilities. We don't administer standardized tests to our students, but we do administer state alternate assessment tests. These tests are now administered by computer and they adjust difficulty level based on the way they think the student is interacting and performing. Last week, I was working with a student who is able to read and has basic reading comprehension, but the computer/program kept adjusting back to object-level tasks. It misjudged her skill level repeatedly and kept offering tasks that were so far below her skill level that it was juvenile and disrespectful. AI Identity: the computer

Human Version: I am a special education teacher working with students with severe disabilities. We don't administer standardized tests to our students, but we do administer state alternate assessment tests. These tests are now administered by a teacher from another school and they adjust difficulty level based on the way they think the student is interacting and performing. Last week, I was working with a student who is able to read and has basic reading comprehension, but the other teacher kept adjusting back to object-level tasks. It misjudged her skill level repeatedly and kept offering tasks that were so far below her skill level that it was juvenile and disrespectful. Human Identity: the teacher from the other school

\section{Strange Chess Moves Scenario}


AI Version: I was playing a chess game with an artificial intelligence. During the middle of the game, I noticed the AI was making strange moves that would have been considered cheating if I was playing with a regular human. I'm not sure if it was a bug in the programming, but I felt cheated during the experience. I ended up switching to different software after. $\boldsymbol{A I}$ Identity: the AI opponent

Human Version: I was playing a chess game against another live person online. During the middle of the game, I noticed the other person was making strange moves that would have been considered cheating in a live game. I'm not sure if it was a miscommunication, but I felt cheated during the experience. I ended up switching to different opponent after. Human Identity: the person online

\section{Voice Memo Scenario}

AI Version: My sister-in-law has a home assistant that she speaks to and gives commands to, and the assistant will do the task. My sister-in-law asked the assistant to record a memo about what she was doing throughout the day. The computer assistant recorded the memo. Then after she was done, she asked the computer assistant to email it to one specific person. Instead, the computer assistant sent the memo to her whole email contact list. As a result, people listened to something they were not supposed to. AI Identity: the computer-based home assistant

Human Version: My sister-in-law has a personal assistant, another real person, that will do tasks for her. She asked the assistant to record a memo about what she was doing throughout the day. The assistant recorded the memo. Then after she was done, she asked the assistant to email it to one specific person. Instead, the assistant sent the memo to her whole email contact list. As a result, people listened to something they were not supposed to. Human Identity: the human personal assistant 